\title{
Profile of Bile Acid Metabolomics in the Follicular Fluid of PCOS Patients
}

\author{
Xiao Yang 1,2,3,4,5 ${ }^{\mathbb{D}}$, Richao Wu ${ }^{1,2,3,4,5}$, Dan Qi 1,2,3,4,5, Linlin Fu 1,2,3,4,5, Tian Song 1,2,3,4,5, Ying Wang 1,2,3,4,5, \\ Yuehong Bian $1,2,3,4,5$ and Yuhua Shi 1,2,3,4,5,*(D)
}

1 Center for Reproductive Medicine, Cheeloo College of Medicine, Shandong University, Jinan 250012, China; yangxiaosduivf@163.com (X.Y.); Wurichao1996@163.com (R.W.); qidan971215@foxmail.com (D.Q.); fulinlin0119@163.com (L.F.); songtian916@163.com (T.S.); 18663708813@163.com (Y.W.); aa19810603@163.com (Y.B.)

2 Key Laboratory of Reproductive Endocrinology of Ministry of Education, Shandong University, Jinan 250012, China

3 Shandong Key Laboratory of Reproductive Medicine, Jinan 250012, China

4 Shandong Provincial Clinical Research Center for Reproductive Health, Jinan 250012, China

5 National Research Center for Assisted Reproductive Technology and Reproductive Genetics, Shandong University, Jinan 250012, China

* Correspondence: shiyuhua2003@126.com

Citation: Yang, X.; Wu, R.; Qi, D.; Fu, L.; Song, T.; Wang, Y.; Bian, Y.; Shi, Y. Profile of Bile Acid Metabolomics in the Follicular Fluid of PCOS Patients. Metabolites 2021, 11, 845. https:// doi.org/10.3390/metabo11120845

Academic Editor: Jennifer W. Hill

Received: 27 October 2021

Accepted: 4 December 2021

Published: 6 December 2021

Publisher's Note: MDPI stays neutral with regard to jurisdictional claims in published maps and institutional affiliations.

Copyright: (c) 2021 by the authors. Licensee MDPI, Basel, Switzerland. This article is an open access article distributed under the terms and conditions of the Creative Commons Attribution (CC BY) license (https:// creativecommons.org/licenses/by/ $4.0 /)$.
Abstract: Polycystic ovary syndrome (PCOS) is a complex heterogeneous endocrine disease affected by genetic and environmental factors. In this manuscript, we aimed to describe the composition of bile acid metabolomics in the follicular fluid (FF) of PCOS. The FF was collected from 31 control patients and 35 PCOS patients diagnosed according to the Rotterdam diagnostic criteria. The Bile Acid Assay Kit and ultra-performance liquid chromatography/tandem mass spectrometry (UPLCMS/MS) were used in this study to detect the total bile acid and 24 bile acid metabolites. Glycocholic acid (GC3A), taurocholic acid (TCA), glycochenodeoxycholic acid (GCDCA), and chenodeoxycholic acid-3- $\beta$-d-glucuronide (CDCA-3Gln) were elevated in the PCOS group. GCDCA was positively correlated with the serum follicle-stimulating hormone (FSH) $(r=0.3787, p=0.0017)$ and luteinizing hormone $(\mathrm{LH})(\mathrm{r}=0.2670, p=0.0302)$. The level of CDCA-3Gln also rose with the increase in antral follicle counts (AFC) $(r=0.3247, p=0.0078)$. Compared with the control group, the primary bile acids $(p=0.0207)$ and conjugated bile acids $(p=0.0283)$ were elevated in PCOS. For the first time, our study described the changes in bile acid metabolomics in the FF of PCOS patients, suggesting that bile acids may play an important role in the pathogenesis of PCOS.

Keywords: polycystic ovary syndrome; bile acids metabonomic; follicular fluid; ovarian micro-environment

\section{Introduction}

Polycystic ovary syndrome (PCOS) is a complex endocrine and metabolic disorder, and is one of the main causes of anovulatory infertility. According to different diagnostic criteria, the prevalence of PCOS in women of childbearing age is approximately 6 20\% [1]. In addition to suffering from infertility, women with PCOS often experience metabolic disorders, and they have an increased risk of type 2 diabetes and cardiovascular disease in the long term [2-5]. Therefore, in order to reduce the psychological burden and social pressure of PCOS patients, many researchers have devoted themselves to revealing the pathogenesis of PCOS and seeking better diagnosis and treatment strategies for patients.

Follicular fluid (FF) is composed of plasma components, follicle secretions, and secretory components of other ovarian cells (ovarian granulosa cells and theca cells), which regulates the micro-environment of the ovary and affects the occurrence and development of oocytes. An important pathophysiological change in PCOS is the increase in the number 
of antral follicles, causing sparse ovulation, which usually manifests as irregular menstruation in the clinic. The pathogenesis of PCOS is usually accompanied by the disorders of proliferation and apoptosis in ovarian granulosa cells [6,7]. Compared with the FF of patients without PCOS, the FF of PCOS patients had higher androgen levels and lower estrogen levels [8]. The alteration of the follicular micro-environment could affect the proliferation of granulosa cells and the arrest of oocytes [9].

Bile acid is a constituent of bile synthesized by the liver and is related to glucose and lipid metabolism. Primary bile acid is biosynthesized in the liver and then decomposed by intestinal flora in the intestine into secondary bile acids. According to whether bile acids are combined with glycine or taurine, they are divided into conjugated bile acids and unconjugated bile acids. In addition to regulating their own synthesis, bile acids have an endocrine function [10]. In recent years, many studies have been devoted to revealing the possible role of bile acids in the pathogenesis of PCOS [11]. In serum, there is a positive correlation with increased circulating conjugated primary bile acids and hyperandrogenemia in PCOS patients [12]. However, there is still no research on bile acid metabolites in the ovary micro-environment of PCOS.

In this manuscript, we reported 24 bile acid metabolites detected by ultra-performance liquid chromatography/tandem mass spectrometry (UPLC-MS/MS) in the FF of PCOS patients and performed quantitative analysis. For the first time, we focused on the bile acid metabolomics of FF in PCOS patients, revealed the differences in bile acid metabolites in the FF of PCOS patients, and suggested that they might play a role in the ovarian micro-environment, in order to provide new insight for the pathogenesis and the clinical treatment of PCOS.

\section{Results}

\subsection{Characteristics of the Participants}

The clinical characteristics of all participants (31 control patients and 35 patients with PCOS) involved in this study were summarized in Table 1. As shown in Table 1, there were no significant differences in aspartate transferase (AST), alanine transferase (ALT), gamma-glutamyl transferase (GGT), triglycerides (TG) or blood glucose levels between control patients and PCOS patients, which excluded the influence of liver function on the state of bile acids in FF. The differences in follicle-stimulating hormone (FSH) levels, body mass index (BMI) and the age between the two groups were also not statistically significant. Statistically significant differences in antral follicle counts (AFC) and the serum levels of anti-Mullerian hormone (AMH), luteinizing hormone (LH), testosterone (T) existed between the two groups. These differences were consistent with the clinical characteristics of PCOS patients.

Table 1. Clinical characteristics between polycystic ovary syndrome (PCOS) group and control group.

\begin{tabular}{cccc}
\hline & Control $(\boldsymbol{n}=\mathbf{3 1})$ & PCOS $(\boldsymbol{n}=\mathbf{3 5})$ & $p$ Value \\
\hline Age (years) & $31.03 \pm 3.95$ & $29.23 \pm 3.84$ & 0.064 \\
AFC (counts) & $14.77 \pm 5.61$ & $27.97 \pm 11.02$ & $p<0.001$ \\
AMH (ng/mL) & $3.50 \pm 1.60$ & $8.40 \pm 4.48$ & $p<0.001$ \\
LH (IU/L) & $5.98 \pm 1.95$ & $11.73 \pm 6.89$ & $p<0.001$ \\
FSH (IU/L) & $6.67 \pm 1.38$ & $6.25 \pm 1.63$ & 0.267 \\
T (ng/dL) & $20.76 \pm 9.99$ & $38.13 \pm 22.12$ & $p<0.001$ \\
BMI (kg/m $)$ & $23.14 \pm 2.78$ & $24.29 \pm 3.00$ & 0.113 \\
Glucose (mmol/L) & $5.26 \pm 0.43$ & $5.17 \pm 0.34$ & 0.345 \\
AST (U/L) & $16.96 \pm 3.75$ & $17.58 \pm 3.20$ & 0.470 \\
ALT (U/L) & $13.64 \pm 5.29$ & $16.45 \pm 7.85$ & 0.096 \\
GGT (U/L) & $13.20 \pm 4.36$ & $16.06 \pm 8.05$ & 0.083 \\
TG (mmol/L) & $0.92 \pm 0.39$ & $0.98 \pm 0.43$ & 0.497 \\
QUICKI & $0.56 \pm 0.09$ & $0.51 \pm 0.11$ & 0.112 \\
\hline AFC, antral follicl
\end{tabular}

$\overline{\mathrm{AFC}}$, antral follicle counts; AMH, anti-Mullerian hormone; LH, luteinizing hormone; FSH, follicle-stimulating hormone; T, testosterone; BMI, body mass index; AST, aspartate transferase; ALT, alanine transferase; GGT, gamma-glutamyl transferase; TG, triglycerides; QUICKI = 1/[log(fasting plasma insulin) + log(fasting plasma glucose). 


\subsection{Differences of Total Bile Acids in Follicular Fluid}

Compared with the control group, the level of total bile acid showed an increasing trend in FF from the PCOS group, but there was no statistically significant difference $(p=0.0640)$ (Figure 1).

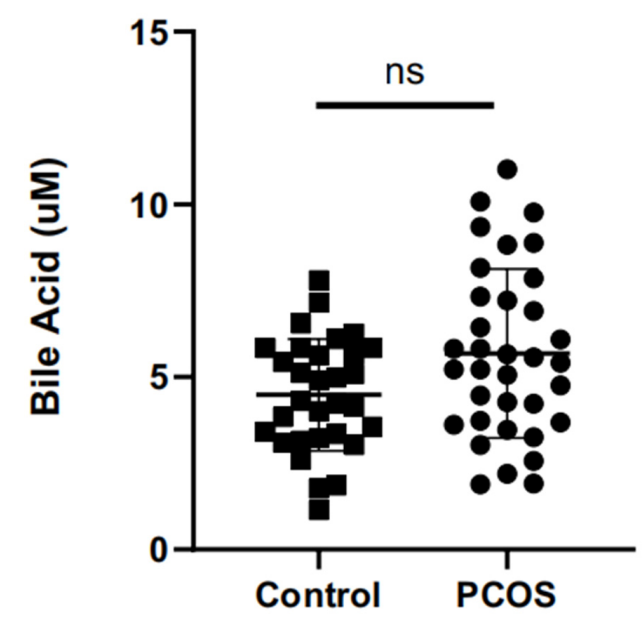

Figure 1. The differences in total bile acids in follicular fluid (FF). ns = not statistically significant.

\subsection{Expression Profile of Bile Acid Metabolites}

In this study, a total of 24 bile acid metabolites were identified by UPLC-MS/MS (Figure 2). Among them, four different bile acid metabolites were detected as being elevated in the PCOS group: GCA $(p=0.0088)$, TCA $(p=0.0302)$, GCDCA $(p=0.0380)$, and CDCA-3Gln $(p=0.0489)$ (Table 2$)$. Furthermore, after further analysis of the bile acid metabolite detection results, it was found that primary bile acids increased significantly in the PCOS group $(p=0.0207)$. However, there was no significant difference in the levels of secondary bile acids in FF between the two groups $(p=0.3062)$. Meanwhile, conjugated bile acids were elevated in PCOS FF $(p=0.0283)$. However, the concentration of unconjugated bile acids was similar between the two groups $(p=0.3778)$.

Table 2. Expression profile of bile acid metabolites in follicular fluid between the two groups.

\begin{tabular}{lccc}
\hline Bile Acid Metabolites (nmol/L) & Control $(\boldsymbol{n}=\mathbf{3 1})$ & PCOS $(\boldsymbol{n}=35)$ & $p$ Value \\
\hline GCA $\#$ & $69.3455(35.0402-108.194)$ & $93.0293(60.9071-139.1637)$ & 0.0088 \\
TCA $\#$ GCDCA \# & $8.9051(6.1289-18.8495)$ & $20.0173(10.2183-32.6538)$ & 0.0302 \\
CDCA-3Gln $\#$ & $813.247(567.252-1181.14)$ & $1045.532(813.6052-1415.405)$ & 0.0380 \\
TUDCA & $2.0029(1.4314-4.7204)$ & $4.5151(1.8605-16.4768)$ & 0.0489 \\
TCDCA & $1.1045(0.4843-3.7344)$ & $1.7891(0.4667-5.5518)$ & 0.7018 \\
TDCA & $101.073(58.0077-132.758)$ & $131.5096(87.4559-197.0543)$ & 0.0586 \\
HCA & $17.21(6.3327-36.5325)$ & $19.6411(4.5489-42.3435)$ & 0.6570 \\
CA & $6.5258(4.3054-11.0712)$ & $7.5380(4.2251-12.5456)$ & 0.8183 \\
NorCA & $39.3982(27.9622-56.5257)$ & $41.8243(29.0361-67.7721)$ & 0.5742 \\
GHCA & $1.3382(0.8645-2.2769)$ & $1.5677(1.0934-2.4134)$ & 0.3376 \\
GUDCA & $6.3761(3.6203-11.9017)$ & $7.7032(5.1268-12.2494)$ & 0.4282 \\
GDCA & $137.575(76.6138-343.196)$ & $181.9499(77.9537-314.5836)$ & 0.9086 \\
GLCA & $88.5226(54.237-145.261)$ & $92.7559(36.1559-218.6125)$ & 0.5484 \\
bUDCA & $3.9102(0.8078-9.2406)$ & $3.3065(0.6672-13.6760)$ & 0.7278 \\
UDCA & $50.0141(30.1774-96.4139)$ & $48.4582(24.3200-105.7436)$ & 0.8985 \\
CDCA & $40.5876(24.5589-65.4812)$ & $40.5971(19.3277-75.6631)$ & 0.8382 \\
DCA & $352.781(177.59-457.672)$ & $375.8702(218.3674-594.6697)$ & 0.3123 \\
LCA & $115.298(55.4131-182.886)$ & $112.2262(34.3780-209.7214)$ & 0.7497 \\
LCA-3S & $2.7428(1.2261-3.8918)$ & $1.9536(0.8700-4.8796)$ & 0.5399 \\
6-KetoLCA & $1.5409(0.3816-2.4319)$ & $0.9994(0.1386-3.2181)$ & 0.6923 \\
7-KetoLCA & $39.4591(30.957-53.853)$ & $35.8543(27.1566-47.3019)$ & 0.3311 \\
12-KetoLCA & $4.1440(2.8514-7.435)$ & $3.8017(2.1842-6.8645)$ & 0.3376 \\
3-DHCA & $3.1705(1.1653-6.6363)$ & $1.9183(0.5242-6.5477)$ & 0.7208 \\
\hline
\end{tabular}

\footnotetext{
\# Statistical significance.
} 
a

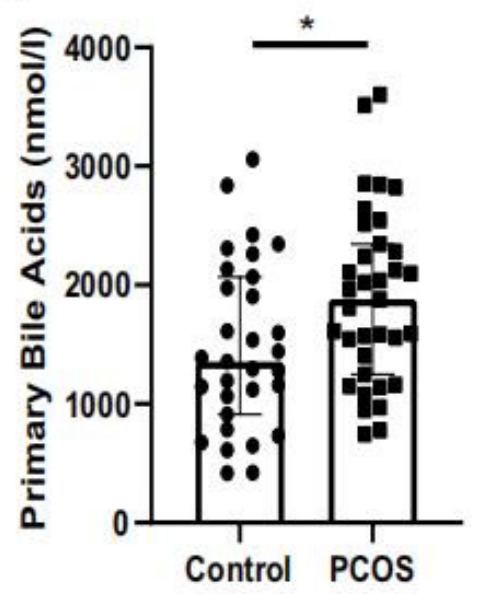

c

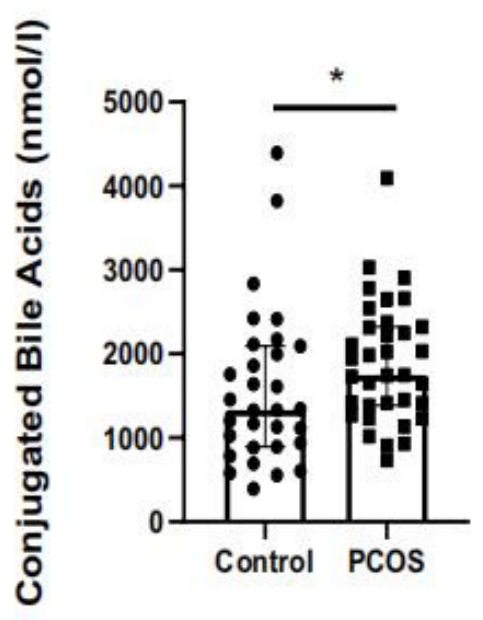

b

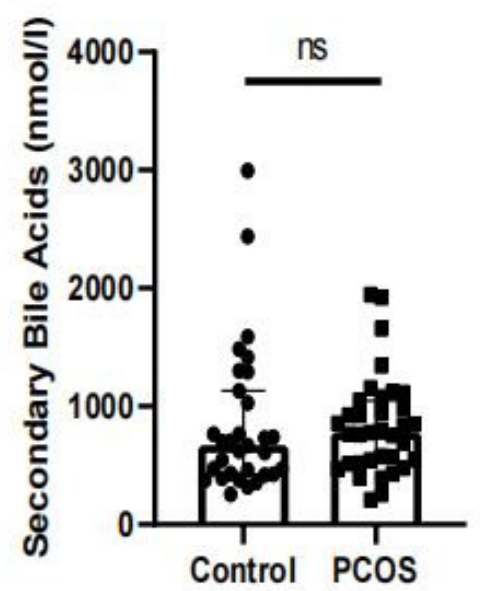

d

อิ $\mathrm{e}$

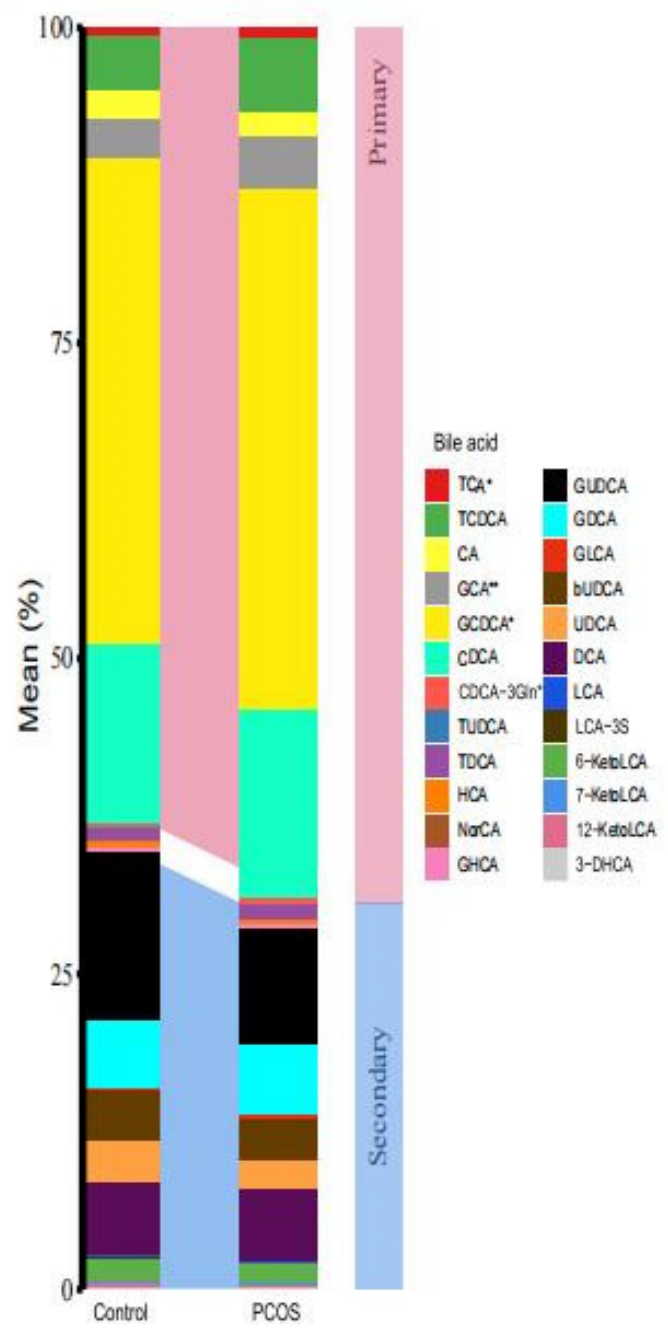

Figure 2. (a) Differences in primary bile acids. (b) Differences in secondary bile acids. (c) Differences in conjugated bile acids. (d) Differences in unconjugated bile acids between the control group and the PCOS group (e) Expression profile of bile acid metabolites in follicular fluid (FF). ${ }^{*} p<0.05,{ }^{* *}$ : Statistical significance; ns = not statistically significant.

\subsection{Correlation between Bile Acid Metabolites and Clinical Characteristics}

After analyzing the correlation between the different quantitative results of bile acid metabolites in FF and the clinical characteristics of the patient, it was found that GCDCA was positively correlated with the serum FSH $(r=0.3787, p=0.0017)$ and LH $(r=0.2670$, $p=0.0302)$. The level of CDCA-3Gln also rose with the increase in AFC $(\mathrm{r}=0.3247, p=0.0078)$. There was no statistical correlation between the analysis of other bile acid metabolites and clinical indicators (Table 3). 
Table 3. The correlation between bile acid metabolites and clinical characteristics.

\begin{tabular}{|c|c|c|c|}
\hline Bile Acid & Clinical Characteristic & Pearson's r & $p$ Value \\
\hline \multirow[t]{7}{*}{ TCA } & $\mathrm{AMH}$ & 0.0922 & 0.4617 \\
\hline & FSH & 0.0400 & 0.7499 \\
\hline & $\mathrm{LH}$ & 0.0898 & 0.4735 \\
\hline & E2 & 0.0286 & 0.8197 \\
\hline & $\mathrm{T}$ & 0.2348 & 0.0577 \\
\hline & AFC & 0.1158 & 0.3546 \\
\hline & QUICKI & 0.0050 & 0.6166 \\
\hline \multirow[t]{7}{*}{ GCA } & $\mathrm{AMH}$ & 0.1590 & 0.2023 \\
\hline & FSH & 0.2027 & 0.1026 \\
\hline & $\mathrm{LH}$ & 0.1123 & 0.3695 \\
\hline & E2 & 0.0475 & 0.7051 \\
\hline & $\mathrm{T}$ & 0.2000 & 0.1074 \\
\hline & AFC & 0.1408 & 0.2594 \\
\hline & QUICKI & 0.0105 & 0.4649 \\
\hline \multirow[t]{7}{*}{ GCDCA } & $\mathrm{AMH}$ & 0.1424 & 0.2514 \\
\hline & $\mathrm{FSH}^{\#}$ & 0.3787 & 0.0017 \\
\hline & $\mathrm{LH}^{\#}$ & 0.2670 & 0.0302 \\
\hline & E2 & 0.1129 & 0.3669 \\
\hline & $\mathrm{T}$ & -0.0001 & 0.9991 \\
\hline & AFC & 0.1481 & 0.2353 \\
\hline & QUICKI & 0.0132 & 0.4125 \\
\hline \multirow[t]{7}{*}{ CDCA-3Gln } & $\mathrm{AMH}$ & 0.2078 & 0.0940 \\
\hline & FSH & -0.1059 & 0.3976 \\
\hline & $\mathrm{LH}$ & 0.0869 & 0.4878 \\
\hline & E2 & 0.0170 & 0.8921 \\
\hline & $\mathrm{T}$ & 0.1655 & 0.1841 \\
\hline & $\mathrm{AFC}^{\#}$ & 0.3247 & 0.0078 \\
\hline & QUICKI & 0.0021 & 0.7466 \\
\hline
\end{tabular}

\# Statistical significance; quantitative insulin sensitivity check index (QUICKI).

\section{Discussion}

In this study, we found that the total bile acid in the FF of PCOS patients was not statistically different from that of the control group, but four different bile acid metabolites were screened out as biomarkers. GCA, TCA, GCDCA and CDCA-3Gln were elevated in the FF of PCOS. Compared with the control group, the primary bile acids in the FF of the PCOS group were significantly increased, which was consistent with previous reports on the PCOS serum bile acid profile [12]. GCA, TCA and GCDCA are conjugated bile acids. Additionally, conjugated bile acids were elevated in the PCOS group significantly. After analyzing the correlation between the different bile acid metabolites and the clinical characteristics of the included patients, it was found that GCDCA was positively correlated with the levels of FSH and LH in serum. It is well known that the LH signal was closely related to the occurrence of ovulation [13]. This result implies that the elevated of GCDCA in FF is related to the ovulation disorder in PCOS. Meanwhile, the level of CDCA-3Gln was positively correlated with AFC, which demonstrated that CDCA-3Gln might be related to the ovarian reserve. At the same time, the status of the insulin resistance was assessed by QUICKI. QUICKI did not change with increase in the levels of the four different bile acid metabolites, which suggests that there was no relationship between the insulin resistance and the level of bile acid metabolites in FF. The present results suggest that certain types of bile acid metabolite may affect the ovarian micro-environment of PCOS patients, which may be related to the growth and development of follicular and ovulation disorders.

As for the four different bile acid metabolites detected, in the China Cardiometabolic Disease and Cancer Cohort (4C) Study, it was found that plasma GCA, TCA, and GCDCA were all related to the increased risk of type 2 diabetes mellitus (T2DM) [14]. In the study of hepatocellular carcinoma (HCC), GCA has been identified as a sensitive biomarker which 
was elevated in the serum and urine of HCC $[15,16]$. GCDCA induces stemness through the STAT3 pathway, thereby promoting the chemoresistance of HCC, it could become a potential target of HCC chemotherapy [17]. TCA promoted the cellular cellularity of retinal pigment epithelial (RPE) cells and reduced choroidal endothelial cell migration and tube formation caused by vascular endothelial growth factor (VEGF) [18].

In previous research, the presence of bile acid metabolites was found in the ovaries of rats [19]. A report described that all aspects of the bile acid synthesis pathway were present in follicles, and proposed that bile acid is produced by granulosa cells [20]. The level of bile acid in human FF was almost twice that of serum, and an increase in the UDCA derivative level was found in FF, resulting in high-quality embryos [21]. Then, the study of Nagy R. A. et al. suggested that the regulation of bile acid transport from blood to FF may have potential effects on the reproductive system [22]. Bile acids are cytotoxic, and it has been proven that excessive bile acids can impair ovarian function by damaging mitochondrial function and inducing oxidative stress [23]. Differences in bile acid metabolites were found between healthy follicles and atretic follicles of Bama Xiang pigs, suggesting that there may be a disorder of bile acid metabolism in porcine atresia follicles [24].

Ovarian granulosa cells could regulate the growth and development of follicles. The presence of farnesoid X receptor (FXR), which serves as the natural receptor of bile acid, has been confirmed in ovarian granulosa cells, and it may play a role in regulating the function of granular cells, thereby affecting ovarian function [25]. As an essential part of bile acid metabolism, liver receptor homolog 1 (LRH-1) also has a role in gonadal development and ovulation $[26,27]$. In our previous study, we discovered high LRH-1 expression in the ovarian granulosa cells of PCOS patients [28]. All this evidence indicates that bile acid metabolites are likely to play a role in follicular development and ovarian function in PCOS. Researchers have found that patients with PCOS have an altered intestinal bile acid metabolism [11]. In the research into serum metabolomics mass spectrometry analysis of PCOS patients, GCA was selected as a biomarker, suggesting that bile acid metabolism may play a role in PCOS [29]. We screened GCA among the differential bile acid metabolites in FF, which was confirmed with the results of the serum.

Previous research only focused on the circulating changes in bile acid in PCOS. In our research, we revealed the differences in bile acid metabolites in the FF of PCOS patients. This implied that bile acid metabolites might affect the regulation of the function of ovarian cells and promote the progression of ovulation disorders in PCOS patients.

\section{Materials and Methods}

\subsection{Participants}

In this study, PCOS was diagnosed according to the Rotterdam criteria (Rotterdam, 2004). Based on the Rotterdam criteria, the diagnosis of PCOS requires at least two of the following three points: oligo- and/or anovulation; clinical and/or biochemical evidence of hyperandrogenism; and ovary polycystic morphology under ultrasound. Other diseases that may cause hyperandrogenism and ovulation disorders need to be excluded, such as Cushing's syndrome, congenital adrenal hyperplasia, and androgen-secreting tumors. The females in control group were those who sought fertility treatment due to oviduct factors or male factors, and they all had regular menstrual cycles. The androgen factor was excluded in the control group. On days 2-5 of the menstrual cycle, with the basal endocrine state, control patients' serum FSH $<10 \mathrm{IU} / \mathrm{L}$ and AMH $>1.5 \mathrm{ng} / \mathrm{mL}$. On the day of using human chorionic gonadotropin (hCG), the follicle count was greater than 5 and less than 15 , to ensure the ovarian reactivity in the control group. The study included 35 patients with PCOS and 31 control patients. All participants were younger than 40 years old and they underwent intracytoplasmic sperm injection (ICSI) or in vitro fertilization-embryo transfer (IVF-ET) treatment in the Reproductive Hospital of Shandong University from July 2020 to November 2020. With the guidance of experienced clinicians, a standard long agonist protocol or antagonist protocol was chosen as the ovulation-stimulation regimen. All patients excluded the history of ovarian surgery, hyperprolactinemia, and thyroid disease. 
According to the guidance of a previous report, the quantitative insulin sensitivity check index $($ QUICKI $)=1 /[\log$ (fasting insulin $)+\log ($ fasting plasma glucose $)]$ was calculated to assess the status of insulin resistance [30]. To eliminate the influence of the liver, all patients were confirmed has having normal liver function. The Ethics Committee of the Affiliated Reproductive Hospital of Shandong University approved this study.

\subsection{The Collection of FF}

After observing proper follicle development through transvaginal ultrasound, clinicians instructed the use of hCG to induce ovulation. After $36 \mathrm{~h}$ of hCG use, during the retrieving of the oocytes, follicular fluid was collected in a sterile centrifuge tube. As quickly as possible, the fresh FF was transported to the laboratory, and centrifuged at $2000 \mathrm{rcf}$ for $10 \mathrm{~min}$. Then, $1 \mathrm{~mL}$ of the supernatant of FF was collected and stored at $-80^{\circ} \mathrm{C}$.

\subsection{The Detection of Total Bile Acid}

Before detecting FF with the kit, samples were taken from $-80^{\circ} \mathrm{C}$ and melted on ice in order to maintain more stable characteristics. The Bile Acid Assay Kit (MAK309) was employed to determine the total bile acid in FF based on the protocol provided by the manufacturer.

\subsection{FF Bile Acid Profile Assessment}

Metabo-Profile Inc. (Shanghai, China) performed the comprehensive profiling and quantitation of the bile acid metabolites using previously published methods [31,32]. The bile acid detection kit-BAP Ultra (Metabo-Profile, Shanghai, China) was used to analyze the bile acid profile in the research. All FF samples were qualified after quality control. Based on UPLC-MS/MS, a total of 24 bile acid metabolites listed in Table 4 were quantified. Primary or secondary bile acids, conjugated or unconjugated bile acids are calculated according to the classification.

Table 4. Abbreviations of bile acid metabolomics.

\begin{tabular}{|c|c|c|c|}
\hline Abbreviation & Full Name & $\begin{array}{c}\text { Classification } \\
\text { (Primary/Secondary) }\end{array}$ & $\begin{array}{c}\text { Classification } \\
\text { (Conjugated/Unconjugated) }\end{array}$ \\
\hline TCA & Taurocholic acid & Primary & Conjugated \\
\hline TUDCA & Tauroursodeoxycholic acid & Secondary & Conjugated \\
\hline TCDCA & Taurochenodeoxycholic acid & Primary & Conjugated \\
\hline TDCA & Taurodeoxycholic acid & Secondary & Conjugated \\
\hline HCA & Hyocholic acid & Secondary & Unconjugated \\
\hline CA & Cholic acid & Primary & Unconjugated \\
\hline NorCA & Norcholic acid & Secondary & Unconjugated \\
\hline GHCA & Glycohyocholate & Secondary & Conjugated \\
\hline GCA & Glycocholic acid & Primary & Conjugated \\
\hline GUDCA & Glycoursodeoxycholic acid & Secondary & Conjugated \\
\hline GCDCA & Glycochenodeoxycholic acid & Primary & Conjugated \\
\hline GDCA & Glycodeoxycholic acid & Secondary & Conjugated \\
\hline GLCA & Glycolithocholate & Secondary & Conjugated \\
\hline bUDCA & Isoursodeoxycholic acid & Secondary & Unconjugated \\
\hline UDCA & Ursodeoxycholic acid & Secondary & Conjugated \\
\hline CDCA & Chenodeoxycholic acid & Primary & Unconjugated \\
\hline DCA & Deoxycholic acid & Secondary & Unconjugated \\
\hline LCA & Lithocholic acid & Secondary & Unconjugated \\
\hline LCA_3S & Lithocholic acid 3 sulfate & Secondary & Conjugated \\
\hline 6_ketoLCA & 6-ketolithocholic acid & Secondary & Unconjugated \\
\hline 7_ketoLCA & 7-ketolithocholic acid & Secondary & Unconjugated \\
\hline 12_ketoLCA & 12-ketolithocholic acid & Secondary & Unconjugated \\
\hline 3_DHCA & 3-oxocholic acid & Secondary & Unconjugated \\
\hline CDCA_3Gln & Chenodeoxycholic acid-3- $\beta$-d-glucuronide & Primary & Conjugated \\
\hline
\end{tabular}




\subsection{Statistical Analysis}

Data analysis was performed using SPSS 26.0 (SPSS, Chicago, IL, USA) and GraphPad Prism 8.0 (GraphPad Software, San Diego, CA, USA). A normality test of continuous variables was performed by using Kolmogorov-Smirnov test. Normally distributed variables were analyzed using Student's t-test to determine statistical significance and displayed in the form of mean \pm standard deviation (SD). Non-normally distributed data are displayed in the form of median and quartile, compared using a nonparametric test. The linear association was assessed by using Pearson's correlational analysis. A $p$ value $<0.05$ was considered statistically significant.

\section{Conclusions}

For the first time, our study explored the bile acid metabolomics in the FF of patients with PCOS, highlighting the impact of bile acid metabolite changes in the ovarian microenvironment. However, the individual differences of clinical samples cannot be ignored, and so a further study with an expanded sample is still needed to reveal the role of bile acid metabolism in the ovarian function of PCOS patients.

Author Contributions: X.Y. and Y.S. conceived and designed this study. R.W. and D.Q. collected samples and clinical data; L.F. and T.S. contributed to statistical analysis; Y.B. and Y.W. participated in the discussion and critically revised the article; and X.Y. drafted the manuscript. All authors have read and agreed to the published version of the manuscript.

Funding: This study was supported by National Key R\&D Program of China (2018YFC1003202, 2017YFC1001004) and Taishan scholar project special funds (No. ts201712103).

Institutional Review Board Statement: The study was conducted according to the guidelines of the Declaration of Helsinki, and approved by the Institutional Review Board of the Center for Reproductive Medicine, Shandong University.

Informed Consent Statement: Informed consent was obtained from all subjects involved in the study.

Data Availability Statement: The data and materials during this study are available from the corresponding author on reasonable requests. The data are not publicly available due to data protection regulation law.

Acknowledgments: The authors thank Lei Yan, Jing Hao, Yanjun Zheng and Jiangtao Zhang for their help and all the patients who participated in the study.

Conflicts of Interest: The authors declare no conflict of interest.

\section{References}

1. Escobar-Morreale, H.F. Polycystic ovary syndrome: Definition, aetiology, diagnosis and treatment. Nat. Rev. Endocrinol. 2018, 14, 270-284. [CrossRef] [PubMed]

2. Christian, R.C.; Dumesic, D.A.; Behrenbeck, T.; Oberg, A.L.; Sheedy, P.F., 2nd; Fitzpatrick, L.A. Prevalence and predictors of coronary artery calcification in women with polycystic ovary syndrome. J. Clin. Endocrinol. Metab. 2003, 88, 2562-2568. [CrossRef]

3. Persson, S.; Elenis, E.; Turkmen, S.; Kramer, M.S.; Yong, E.L.; Poromaa, I.S. Higher risk of type 2 diabetes in women with hyperandrogenic polycystic ovary syndrome. Fertil. Steril. 2021, 116, 862-871. [CrossRef] [PubMed]

4. Zhu, T.; Cui, J.; Goodarzi, M.O. Polycystic Ovary Syndrome and Risk of Type 2 Diabetes, Coronary Heart Disease, and Stroke. Diabetes 2021, 70, 627-637. [CrossRef]

5. Paul, C.; Lagana, A.S.; Maniglio, P.; Triolo, O.; Brady, D.M. Inositol's and other nutraceuticals' synergistic actions counteract insulin resistance in polycystic ovarian syndrome and metabolic syndrome: State-of-the-art and future perspectives. Gynecol. Endocrinol. 2016, 32, 431-438. [CrossRef]

6. Tu, J.; Chen, Y.; Li, Z.; Yang, H.; Chen, H.; Yu, Z. Long non-coding RNAs in ovarian granulosa cells. J. Ovarian Res. 2020, 13, 63. [CrossRef]

7. Zhou, L.; Han, X.; Li, W.; Wang, N.; Yao, L.; Zhao, Y.; Zhang, L. N6-methyladenosine Demethylase FTO Induces the Dysfunctions of Ovarian Granulosa Cells by Upregulating Flotillin 2. Reprod. Sci. 2021. ahead of print. [CrossRef] [PubMed]

8. Naessen, T.; Kushnir, M.M.; Chaika, A.; Nosenko, J.; Mogilevkina, I.; Rockwood, A.L.; Carlstrom, K.; Bergquist, J.; Kirilovas, D. Steroid profiles in ovarian follicular fluid in women with and without polycystic ovary syndrome, analyzed by liquid chromatography-tandem mass spectrometry. Fertil. Steril. 2010, 94, 2228-2233. [CrossRef] [PubMed] 
9. Liu, Y.; Liu, H.; Li, Z.; Fan, H.; Yan, X.; Liu, X.; Xuan, J.; Feng, D.; Wei, X. The Release of Peripheral Immune Inflammatory Cytokines Promote an Inflammatory Cascade in PCOS Patients via Altering the Follicular Microenvironment. Front. Immunol. 2021, 12, 685724. [CrossRef]

10. Houten, S.M.; Watanabe, M.; Auwerx, J. Endocrine functions of bile acids. EMBO J. 2006, 25, 1419-1425. [CrossRef]

11. Qi, X.; Yun, C.; Sun, L.; Xia, J.; Wu, Q.; Wang, Y.; Wang, L.; Zhang, Y.; Liang, X.; Wang, L.; et al. Gut microbiota-bile acidinterleukin-22 axis orchestrates polycystic ovary syndrome. Nat. Med. 2019, 25, 1225-1233. [CrossRef]

12. Zhang, B.; Shen, S.; Gu, T.; Hong, T.; Liu, J.; Sun, J.; Wang, H.; Bi, Y.; Zhu, D. Increased circulating conjugated primary bile acids are associated with hyperandrogenism in women with polycystic ovary syndrome. J. Steroid. Biochem. Mol. Biol. 2019, 189, 171-175. [CrossRef] [PubMed]

13. Bianco, S.; Bellefleur, A.M.; Beaulieu, E.; Beauparlant, C.J.; Bertolin, K.; Droit, A.; Schoonjans, K.; Murphy, B.D.; Gevry, N. The Ovulatory Signal Precipitates LRH-1 Transcriptional Switching Mediated by Differential Chromatin Accessibility. Cell Rep. 2019, 28, 2443-2454.e4. [CrossRef] [PubMed]

14. Lu, J.; Wang, S.; Li, M.; Gao, Z.; Xu, Y.; Zhao, X.; Hu, C.; Zhang, Y.; Liu, R.; Hu, R.; et al. Association of Serum Bile Acids Profile and Pathway Dysregulation With the Risk of Developing Diabetes Among Normoglycemic Chinese Adults: Findings from the 4C Study. Am. Diabetes Care 2021, 44, 499-510. [CrossRef] [PubMed]

15. Chen, T.; Xie, G.; Wang, X.; Fan, J.; Qiu, Y.; Zheng, X.; Qi, X.; Cao, Y.; Su, M.; Wang, X.; et al. Serum and urine metabolite profiling reveals potential biomarkers of human hepatocellular carcinoma. Mol. Cell Proteom. 2011, 10, M110.004945. [CrossRef] [PubMed]

16. Zhang, A.; Sun, H.; Yan, G.; Han, Y.; Ye, Y.; Wang, X. Urinary metabolic profiling identifies a key role for glycocholic acid in human liver cancer by ultra-performance liquid-chromatography coupled with high-definition mass spectrometry. Clin. Chim. Acta 2013, 418, 86-90. [CrossRef]

17. Shi, C.; Yang, J.; Hu, L.; Liao, B.; Qiao, L.; Shen, W.; Xie, F.; Zhu, G. Glycochenodeoxycholic acid induces stemness and chemoresistance via the STAT3 signaling pathway in hepatocellular carcinoma cells. Aging 2020, 12, 15546-15555. [CrossRef]

18. Warden, C.; Barnett, J.M.; Brantley, M.A., Jr. Taurocholic acid inhibits features of age-related macular degeneration in vitro. Exp. Eye Res. 2020, 193, 107974. [CrossRef]

19. Gaikwad, N.W. Bileome: The bile acid metabolome of rat. Biochem. Biophys. Res. Commun. 2020, 533, 458-466. [CrossRef]

20. Smith, L.P.; Nierstenhoefer, M.; Yoo, S.W.; Penzias, A.S.; Tobiasch, E.; Usheva, A. The bile acid synthesis pathway is present and functional in the human ovary. PLoS ONE 2009, 4, e7333. [CrossRef]

21. Nagy, R.A.; van Montfoort, A.P.; Dikkers, A.; van Echten-Arends, J.; Homminga, I.; Land, J.A.; Hoek, A.; Tietge, U.J. Presence of bile acids in human follicular fluid and their relation with embryo development in modified natural cycle IVF. Hum. Reprod. 2015, 30, 1102-1109. [CrossRef]

22. Nagy, R.A.; Hollema, H.; Andrei, D.; Jurdzinski, A.; Kuipers, F.; Hoek, A.; Tietge, U.J.F. The Origin of Follicular Bile Acids in the Human Ovary. Am. J. Pathol. 2019, 189, 2036-2045. [CrossRef]

23. Ommati, M.M.; Farshad, O.; Niknahad, H.; Arabnezhad, M.R.; Azarpira, N.; Mohammadi, H.R.; Haghnegahdar, M.; Mousavi, K.; Akrami, S.; Jamshidzadeh, A.; et al. Cholestasis-associated reproductive toxicity in male and female rats: The fundamental role of mitochondrial impairment and oxidative stress. Toxicol. Lett. 2019, 316, 60-72. [CrossRef]

24. Mo, J.; Sun, L.; Cheng, J.; Lu, Y.; Wei, Y.; Qin, G.; Liang, J.; Lan, G. Non-targeted Metabolomics Reveals Metabolic Characteristics of Porcine Atretic Follicles. Front. Vet. Sci. 2021, 8, 679947. [CrossRef]

25. Takae, K.; Nakata, M.; Watanabe, T.; Sasada, H.; Fujii, H.; Tomioka, I. Evidence for the involvement of FXR signaling in ovarian granulosa cell function. J. Reprod. Dev. 2019, 65, 47-55. [CrossRef]

26. Duggavathi, R.; Volle, D.H.; Mataki, C.; Antal, M.C.; Messaddeq, N.; Auwerx, J.; Murphy, B.D.; Schoonjans, K. Liver receptor homolog 1 is essential for ovulation. Genes Dev. 2008, 22, 1871-1876. [CrossRef]

27. Hinshelwood, M.M.; Shelton, J.M.; Richardson, J.A.; Mendelson, C.R. Temporal and spatial expression of liver receptor homologue1 (LRH-1) during embryogenesis suggests a potential role in gonadal development. Dev. Dyn. 2005, 234, 159-168. [CrossRef]

28. Yang, X.; Wang, Q.; Wang, Y.; Song, T.; Zheng, Y.; Wang, W.; Shi, Y. LRH-1 high expression in the ovarian granulosa cells of PCOS patients. Endocrine 2021, 74, 413-420. [CrossRef] [PubMed]

29. Jia, C.; Xu, H.; Xu, Y.; Xu, Y.; Shi, Q. Serum metabolomics analysis of patients with polycystic ovary syndrome by mass spectrometry. Mol. Reprod. Dev. 2019, 86, 292-297. [CrossRef] [PubMed]

30. Katz, A.; Nambi, S.S.; Mather, K.; Baron, A.D.; Follmann, D.A.; Sullivan, G.; Quon, M.J. Quantitative Insulin Sensitivity Check Index: A Simple, Accurate Method for Assessing Insulin Sensitivity In Humans. J. Clin. Endocrinol. Metab. 2000, 85, 2402-2410. [CrossRef] [PubMed]

31. Wang, X.; Xie, G.; Zhao, A.; Zheng, X.; Huang, F.; Wang, Y.; Yao, C.; Jia, W.; Liu, P. Serum Bile Acids Are Associated with Pathological Progression of Hepatitis B-Induced Cirrhosis. J. Proteome Res. 2016, 15, 1126-1134. [CrossRef]

32. Xie, G.; Wang, Y.; Wang, X.; Zhao, A.; Chen, T.; Ni, Y.; Wong, L.; Zhang, H.; Zhang, J.; Liu, C.; et al. Profiling of serum bile acids in a healthy Chinese population using UPLC-MS/MS. J. Proteome Res. 2015, 14, 850-859. [CrossRef] [PubMed] 